\title{
Does smoking marijuana increase the risk of chronic obstructive pulmonary disease?
}

\author{
Donald P. Tashkin MD
}

$\infty$ See related research paper by Tan and colleagues, page 814

$\mathrm{M}$ arijuana is the second most commonly smoked substance worldwide after tobacco. ${ }^{1}$ The constituents of marijuana smoke are qualitatively and, to a large extent, quantitatively similar to those of tobacco smoke, with the exceptions of $\Delta^{9}$-tetrahydrocannabinol (THC), found only in marijuana, and nicotine, found only in tobacco. Given these similarities, there is concern that the health risks of regular marijuana smoking may be similar to those of habitual tobacco smoking. Chronic obstructive pulmonary disease (COPD), which is associated with high morbidity and mortality, is among those risks.

Over the past 2 decades, studies have addressed the possible relation between smoking marijuana and COPD by systematically assessing respiratory symptoms and measuring lung function in smokers and nonsmokers of marijuana or tobacco. $^{2-8}$ These cross-sectional ${ }^{2,3,6}$ and longitudinal ${ }^{4,5,7}$ studies have used convenience sampling, random or stratified random sampling or birth cohorts from the general community. One study examined lung structure using thoracic high-resolution computed tomography to identify macroscopic emphysema. ${ }^{8}$

In this issue of CMAJ, Tan and colleagues report the findings of a cross-sectional population-based study of the possible association between smoking marijuana and risk of COPD. ${ }^{9}$ Whereas previous studies have consistently reported an association between use of marijuana and chronic respiratory symptoms even in the absence of concomitant use of tobacco, ${ }^{2,3,6,8}$ Tan and colleagues did not report a similar finding for marijuana only. However, they found that the concurrent use of marijuana and tobacco appeared to synergistically increase respiratory symptoms and risk of COPD.

An association between using marijuana and COPD has been suggested by evidence of proximal airway injury (i.e., increased erythema, edema and mucous secretions) observed during bronchoscopy in both marijuana-only and tobacco smokers. ${ }^{10}$ Such an association has also been suggested by histopathologic evidence of goblet cell metaplasia, loss of ciliated columnar epithelial cells and inflammatory changes in the bronchial mucosa of smokers of marijuana and smokers of both marijuana and tobacco. ${ }^{10,11}$

The evidence for an association between use of marijuana and abnormalities in lung function, however, is inconsistent. In a convenience sample of young men (mean age 33 years) in Los Angeles, United States, who were habitual, heavy smokers of marijuana or tobacco and nonsmoking controls $(n=446)$, smoking only marijuana was not associated with

\section{Key points}

- Given the increased risk of chronic obstructive pulmonary disease (COPD) among smokers of tobacco, there is concern that a similar risk may exist among smokers of marijuana.

- A limited body of evidence suggests an association between regular marijuana smoking and adverse effects on respiratory health. Studies that address this possibility have many limitations, however.

- The finding by Tan and colleagues that smoking both marijuana and tobacco is associated with a greater risk of COPD than smoking only tobacco suggests an additive effect of the 2 substances on lung health. This study involved an older population that is more at risk of COPD.

- Marijuana smoking by itself probably does not lead to COPD.

abnormalities in forced expiratory volume in 1 second $\left(\mathrm{FEV}_{1}\right)$, forced vital capacity (FVC), ratio of $\mathrm{FEV}_{1}$ to $\mathrm{FVC}$, various measures of small airway function or single-breath diffusing capacity for carbon monoxide. ${ }^{2}$ However, a modest decrease in specific airway conductance was observed in those who smoked only marijuana. This decrease was consistent with endoscopic observations of slight narrowing of the central airways and edematous changes in the tracheobronchial mucosa in this subgroup. ${ }^{10}$ An 8 -year longitudinal extension of this study did not show an age-related accelerated decline in $\mathrm{FEV}_{1}$ among participants who smoked only marijuana compared with participants who did not smoke marijuana or tobacco. There was a significant accelerated decline among those who smoked tobacco.

By contrast, an epidemiologic study in Tucson, United States, involving participants aged $15-40$ years $(n=585)$ found a slight but significant decrease in the ratio of $\mathrm{FEV}_{1}$ to FVC and in a measure of obstruction of the small airways among participants who used marijuana independent of tobacco. ${ }^{3}$ A related longitudinal study in Tucson involving 856 participants aged 15-60 years showed slight decreases in $\mathrm{FEV}_{1}$ and in the ratio of $\mathrm{FEV}_{1}$ to FVC among those who had previously smoked marijuana but not among those who currently smoked marijuana. ${ }^{4}$ In a birth cohort of 1037 participants aged 21 years in Dunedin, New Zealand, a reduced ratio of $\mathrm{FEV}_{1}$ to FVC (using a threshold of $<80 \%$ ) was found in a

Donald Tashkin is with the Division of Pulmonary and Critical Care Medicine, David Geffen School of Medicine, University of California Los Angeles (UCLA), Los Angeles, USA. 
significantly higher percentage of participants who showed signs of marijuana dependency and did not smoke tobacco than in participants who smoked neither marijuana nor tobacco $(36 \%$ v. $20 \%)(p=0.04) .{ }^{6}$ In the same cohort, longitudinal observations of participants up to 26 years of age showed a nonsignificant trend toward a dose-dependent relation between cumulative use of marijuana and a decreased ratio of $\mathrm{FEV}_{1}$ to slow vital capacity after adjustment for smoking tobacco and other covariates $(p=0.082){ }^{7}$ The results of this study also suggested an additive influence of marijuana and daily use of tobacco.

In a convenience sample of 339 residents of Wellington, New Zealand, aged 18-70 years (mean age 43.4) comprising nonsmokers and smokers of either marijuana only, tobacco only or both substances, the authors reported that there was no association between use of marijuana and abnormalities in lung function (including lung volume and diffusing capacity). ${ }^{8}$ However, regression analyses with marijuana as a continuous variable showed significant associations between lifetime cumulative use of marijuana and airflow obstruction (measured by both the ratio of $\mathrm{FEV}_{1}$ to $\mathrm{FVC}$ and specific airway conductance) and between use of marijuana and hyperinflation (measured by total lung capacity). The same study did not show an association between smoking marijuana and evidence of macroscopic emphysema, as shown by high-resolution computed tomography.

These studies, involving mainly younger individuals, have provided inconsistent but suggestive evidence that smoking marijuana only may lead to modest airflow obstruction and hyperinflation that could predispose the individual to COPD later in life. The findings of Tan and colleagues ${ }^{9}$ add to the limited evidence of an association between use of marijuana and COPD because their study focuses on an older population (aged 40 or older) that is at greater risk of COPD. Their finding that concurrent smoking of marijuana and tobacco is associated with a greater likelihood of COPD than smoking only tobacco implies a possible additive effect of the 2 substances on lung health. An additive effect was also suggested by Taylor and colleagues, ${ }^{7}$ whose study involved a younger population. By contrast, Aldington and colleagues ${ }^{8}$ concluded that concurrent smoking of marijuana and tobacco attenuated the association between smoking tobacco and a reduced ratio of $\mathrm{FEV}_{1}$ to $\mathrm{FVC}$ and respiratory symptoms.

Firm conclusions cannot be drawn about the association between use of marijuana and COPD based on the limited and inconsistent data available. The studies that address this topic are limited by their small numbers of participants and by the uncertain accuracy of self-reported use of marijuana, particularly in view of its illegality and the difficulty of accurately recalling amounts previously used. Some of these studies are also limited by their cross-sectional design, and most are limited by the young age (40 years or younger) of participants. Nevertheless, the consistency of some aspects of the available data allows us to more firmly conclude that smoking marijuana by itself can lead to respiratory symptoms because of injurious effects of the smoke on larger airways. Given the consistently reported absence of an association between use of marijuana and abnormal diffusing capacity or signs of macroscopic emphysema, we can be close to concluding that smoking marijuana by itself does not lead to COPD.

Competing interests: None declared.

\section{REFERENCES}

1. World drug report 2006. New York (NY): United Nations Office on Drugs and Crime; 2006. Available: www.unodc.org/pdf/WDR_2006/wdr2006_volume1.pdf (accessed 2009 Mar. 18)

2. Tashkin DP, Coulson AH, Clark VA, et al. Respiratory symptoms and lung function in habitual, heavy smokers of marijuana alone, smokers of marijuana and tobacco, smokers of tobacco alone, and nonsmokers. Am Rev Respir Dis 1987;135: 209-16.

3. Bloom JW, Kaltenborn WT, Paoletti P, et al. Respiratory effects of non-tobacco cigarettes. BMJ 1987;295:1516-8.

4. Sherrill DL, Krzyzanowski M, Bloom JW, et al. Respiratory effects of non-tobacco cigarettes: a longitudinal study in general population. Int J Epidemiol 1991;20:132-7.

5. Tashkin DP, Simmons MS, Sherrill DL, et al. Heavy habitual marijuana smoking does not cause an accelerated decline in $\mathrm{FEV}_{1}$ with age. Am J Respir Crit Care Med 1997; 155:141-8.

6. Taylor DR, Poulton R, Moffitt TE, et al. The respiratory effects of cannabis dependence in young adults. Addiction 2000;95:1669-77.

7. Taylor DR, Fergusson DM, Milne BJ, et al. A longitudinal study of the effects of tobacco and cannabis exposure on lung function in young adults. Addiction 2002; 97:1055-61.

8. Aldington S, Williams M, Nowitz M, et al. Effects of cannabis on pulmonary structure, function and symptoms. Thorax 2007;62:1058-63.

9. Tan WC, Lo C, Jong A, et al.; for the Vancouver Burden of Obstructive Lung Disease (BOLD) Research Group. Marijuana and chronic obstructive lung disease: a population-based study. CMAJ 2009;180:814-20.

10. Roth MD, Arora A, Barsky SH, et al. Airway inflammation in young marijuana and tobacco smokers. Am J Respir Crit Care Med 1998;157:928-37.

11. Fligiel SE, Roth MD, Kleerup EC, et al. Tracheobronchial histopathology in habitual smokers of cocaine, marijuana, and/or tobacco. Chest 1997;112:319-26.

Correspondence to: Dr. Donald P. Tashkin, Department of

Medicine, David Geffen School of Medicine, University of

California Los Angeles (UCLA), 10833 Le Conte Ave.,

Los Angeles CA 90095-1690, USA; fax 310 206-5088;

dtashkin@mednet.ucla.edu 